\title{
Reasons for not having received influenza vaccination and its predictors in Canadians
}

\author{
This article was published in the following Dove Press journal: \\ Vaccine: Development and Therapy \\ 10 August 2012 \\ Number of times this article has been viewed
}

\author{
Yue Chen' \\ Jun $\mathrm{Wu}^{2}$ \\ Qi-long $\mathrm{Yi}^{1}$ \\ Julie Laroche ${ }^{3}$ \\ Thomas Wong ${ }^{2}$ \\ 'Department of Epidemiology and \\ Community Medicine, Faculty of \\ Medicine, University of Ottawa, \\ 2Professional Guidelines and Public \\ Health Practice Division, Centre \\ for Communicable Diseases and \\ Infection Control, Public Health \\ Agency of Canada, ${ }^{3}$ mmunization \\ Assessment and Information, Centre \\ for Immunization and Respiratory \\ Infectious Diseases, Public Health \\ Agency of Canada, Ottawa, Ontario, \\ Canada
}

Background: Influenza vaccination is the most effective way to prevent influenza. However, only about one-third of Canadians receive an annual seasonal influenza vaccination.

Methods: The reasons for not having received influenza vaccination were examined among 131,061 Canadians $\geq 12$ years of age who participated in a national survey in 2007-2008. Among them, 127,297 subjects responded to the questions concerning their flu shot history and were grouped into three categories: never $(n=51,767), 1+$ year ago $(n=29,310)$, last year ( $n=46,220$ ). Subjects who reported not having had a flu shot during the past year were asked the reasons for not having it. The log binomial regression model was used to estimate prevalence ratios (PRs) and 95\% confidence intervals (95\% CIs) for the associations of various reasons for not having received influenza vaccination and their predictors.

Results: When weighted to the Canadian population, $44.0 \%$ had never previously received influenza vaccine and $24.5 \%$ had received the vaccine $>12$ months ago. The most common reasons for not having received influenza vaccination in the past 12 months were "Respondent did not think it necessary" (71.3\%) and "Have not gotten around to it" (17.6\%). Log binomial regression analysis shows that females were less likely to report these two reasons compared to males with PRs of $0.98(0.97,0.99)$ and $0.84(0.81,0.87)$, respectively. Younger participants were more likely to report, "Have not gotten around to it." For those who had an influenza vaccination previously, the primary reason for not having an influenza vaccination in the last year was "Have not gotten around to it."

Conclusions: More than two-thirds of Canadians 12+ years of age did not receive an influenza vaccination in the past year, and "Respondent did not think it necessary" and "Have not gotten around to it" were the main reasons.

Keywords: Canada, flu shot, human, influenza, survey, vaccination

\section{Introduction}

Seasonal influenza is an important public health concern and vaccination against seasonal influenza is an effective method for prevention. ${ }^{1}$ Influenza vaccination reduces the public health burden for influenza and complications ${ }^{2}$ and has been found to be associated with reductions in all-cause mortality, ${ }^{3}$ hospitalizations, ${ }^{4}$ emergency room visits, ${ }^{5}$ as well as physician visits. ${ }^{6}$ Being vaccinated each year with the seasonal influenza vaccine is the most effective way to prevent influenza. ${ }^{7}$

In spite of the benefits, only about one-third or less of Canadians 12 years of age or older receive an annual seasonal influenza vaccination. ${ }^{8,9}$ Even in Ontario, which has a Universal Influenza Immunization Program, approximately $60 \%$ of residents do not receive the seasonal influenza vaccine. ${ }^{9}$
Correspondence: Yue Chen Department of Epidemiology and Community Medicine, Faculty of Medicine, University of Ottawa, 45I Smyth Road, Ottawa, Ontario, Canada KIH 8 M5 Tel +l 6135625800 ext 8287 Fax + I 6I 35625465 Email ychen@uottawa.ca 
In the Canadian Community Health Survey, which targets the Canadian population aged 12 years or older, the reasons for not having received an influenza vaccination in the past year were examined. The current analysis is based on data collected in 2007-2008, which were collected before the H1N1 pandemic, with an intention to avoid its potential impact on seasonal influenza vaccination coverage. The purpose of the study is to better understand the reasons for not having received a seasonal influenza vaccination and factors related to influenza vaccination status in order to design better influenza vaccination strategies to improve vaccination rates for seasonal influenza in the general population.

\section{Materials and methods Study population}

In the present study, we used data from the Canadian Community Health Survey (CCHS) conducted by Statistics Canada in 2007 and 2008. The CCHS is "a cross-sectional survey that collects information related to health status, health care utilization and health determinants for the Canadian population" (Canadian Community Health Survey - Annual Components. User Guide. 2007-2008 microdata file. Statistics Canada, Health Statistics Division). Household residents aged 12 years or more in all the ten provinces and three territories in Canada were targeted in the survey, excluding individuals living on Indian Reserves or Crown lands, residents of institutions, full-time members of the Canadian Armed Forces, and residents in certain remote regions. This survey used a multistage stratified sampling design and three sampling frames to select the sample of households: an area frame of the Canadian Labour Force Survey, a list frame of telephone numbers, and a Random Digit Dialing (RDD) sampling frame. The publicly released data file includes a total of 131,061 persons 12 years of age or more, and the survey response rate was $76.4 \%$, which has been described in detail in the CCHS User Guide.

\section{Questionnaire administration and main outcomes}

The questionnaire was administered using computer-assisted interviewing. Sampling units selected from a telephone list frame were interviewed from centralized centers, and those selected from an area frame were interviewed mainly by decentralized field interviewers. The analysis was based on data from 127,297 subjects who responded to the question, "Have you ever had a flu shot?," and who further responded to the question, "When did you have your last flu shot: less than 1 year ago, 1 year to less than 2 years ago, or 2 years ago or more?" Subjects were grouped into three categories based on their flu shot history: never $(n=51,767), 1+$ year ago $(n=29,310)$, and last year $(n=46,220)$. Subjects who reported not having had a flu shot during the past year were asked, "What are the reasons that you have not had a flu shot in the past year?" One or more choices could be selected by the respondent, including: (1) Have not gotten around to it; (2) Respondent did not think it was necessary; (3) Doctor did not think it was necessary; (4) Personal or family responsibilities; (5) Not available at time required; (6) Not available at all in the area; (7) Waiting time was too long; (8) Transportation problems; (9) Language problem; (10) Cost; (11) Did not know where to go/uninformed; (12) Fear (eg, painful, embarrassing, find something wrong); (13) Bad reaction to previous shot; (14) Unable to leave the house because of a health problem; and 15) Other.

\section{Predictors}

Subjects were asked if the person had "long-term conditions," which were expected to last or had already lasted six months or more and that had been diagnosed by a health professional. Long-term conditions were grouped into two categories. "Major disease" category in the present analysis included asthma, chronic bronchitis/emphysema/ chronic obstructive pulmonary disease (COPD), diabetes, heart disease, cancer, and effects of stroke, which are associated with a high risk of influenza complications. The "nonmajor chronic disease" category included arthritis, back pain, high blood pressure, migraine headaches, stomach and intestinal ulcers, urinary incontinence, bowel disorder, mood disorder, and anxiety disorder. Self-perceived health was categorized by asking the question: "In general, would you say your health is excellent, very good, good, fair, or poor?" Based on total household income, subjects were classified into low- $(<\$ 40,000)$, middle- $(\$ 40,000-\$ 79,999)$, or high-income groups $(\$ 80,000+)$. Subjects were grouped into three education categories: low education (not proceeding beyond secondary school), middle education (secondary school completed with or without some post-secondary education), and high education (post-secondary school certificate or diploma). The "Current smoker" group consisted of respondents who had smoked at least 100 cigarettes during their lifetime and reported smoking cigarettes every day or almost every day at the time of the survey. Former smokers were those who reported smoking cigarettes daily in the past but were not smoking at the time of the survey. Otherwise, subjects were classified as non-smokers. Based on the total daily energy expenditure values ( $\mathrm{kcal} / \mathrm{kg} /$ day), 
the subjects were grouped into three categories: active $(>3)$, moderate active (1.5-2.9), and inactive $(<1.5)$. Energy expenditure (EE) was calculated based on the frequency and duration of leisure-time physical activity (LTPA) and its value of metabolic energy cost, expressed as a multiple of the resting metabolic rate (MET). Other variables included in the analysis were age $(12-24,25-44,45-64,65+)$ and immigrant status (yes or no).

\section{Statistical analysis}

The distribution of socio-demographic factors and health status (major and non-major chronic disease, self-perceived health) were compared among three groups with different influenza immunization status histories. Reasons for not having a flu shot in the past years were compared between those who never had one and those who had one more than one year ago. Each reason in association with sociodemographic factors and health status was investigated for those who had not had a flu shot in the past year. Adjusted relative risks and $95 \%$ confidence intervals for the main reasons for not having a flu shot in the past year in relation to socio-demographic factors and health status were calculated by using log-binomial regression analysis. Model parameters were estimated and tested using the method of maximum likelihood. All of the variance estimates accounted for the multiple stage and stratified survey design. The effect of the complex survey design on variance estimates is summarized as a design effect. The design effect is the ratio of an estimated variance based on the survey to a comparable estimate of variance from a simple random sample of the population. Standard errors were inflated by this average design effect. ${ }^{10}$ First, population weights were divided by the average weight for all subjects included in the analysis. The sum of these relative weights is the effective sample size. Next, we divided the relative weights by the square root of the average design effect. All of the statistical analyses were conducted using SAS 9.2.

\section{Results}

\section{Influenza coverage and population characteristics}

Among the participants, $44.0 \%$ reported never having received a dose of influenza vaccine and $24.5 \%$ had the last one more than 12 months ago. Table 1 shows that participants who were male, younger, or healthier were less likely to report having received influenza vaccination in the past year as compared to those who were female, older, or less healthy. Immigrants and those with lower income tended to have a higher influenza vaccination coverage in the past.

\section{Reasons for not having received influenza vaccination}

The common reasons reported for not having received influenza vaccination included "Respondent did not think it necessary" (71.3\%), "Have not gotten around to it" (17.6\%), "Bad reaction to previous shot" (5.3\%), "Fear" (4.1\%), and "Doctor did not think it necessary" (2.3\%) (Table 2). Only about $1 \%$ or less of the participants reported each of the following reasons: "Personal/family responsibility," "Not available at time when required," "Not available at all in area," "Waiting time was too long," "Transportation problems," "Language problem," "Cost," "Did not know where to go," and "Unable to leave house/health" (Table 2). Middle-aged people (25-64 years) were more likely to think it unnecessary, young adults tended to be more likely to not have gotten around to it, and the elderly were more likely to have had a bad reaction to a previous one (Figure 1).

\section{Predictors of reasons for not having received influenza vaccination}

Log-binomial regression analysis was used to examine various factors associated with the five major reasons for not having received influenza vaccination among the participants who reported having had no flu shot in the past year, taking account for covariates. Table 3 shows that compared with males, females were less likely to report "Respondent did not think it necessary" (PR: 0.98, 95\% CI: 0.97, 0.99) and "Have not gotten around to it" (PR: 0.84, 95\% CI: 0.81, 0.88) and were more likely to report "Bad reaction to previous shot" (PR: 1.33, 95\% CI: 1.23, 1.43), "Fear" (PR: 1.66, 95\% CI: $1.53,1.82$ ), and "Doctor did not think it necessary" (PR: 1.51, 95\% CI: 1.34, 1.70). Young participants were more likely to report, "Have not gotten around to it" and less likely to report other reasons. Smokers were less likely to report, "Doctor did not think it necessary." Whites and active individuals were more likely to report "Bad reaction to previous shot." Having a major or non-major chronic condition or self-perceived poor health were associated with higher proportions of "Bad reaction to previous shot," "Fear," and "Doctor did not think it necessary" but a lower proportion of "Respondent did not think it necessary." Self-perceived health was strongly correlated with having a chronic disease. When chronic disease was excluded from the model, we observed stronger association of self-perceived health and various reasons. Figure 2 shows the relative risk for self-perceived health 
Table I Social-demographic characteristics and health status associated with influenza vaccination history, Canadian Community Health Survey 2007-2008

\begin{tabular}{|c|c|c|c|c|c|c|}
\hline \multirow[t]{3}{*}{ Characteristics } & \multicolumn{6}{|c|}{ Influenza vaccination history } \\
\hline & \multicolumn{2}{|c|}{$\begin{array}{l}\text { Never } \\
(n=5 \mid 767)\end{array}$} & \multicolumn{2}{|c|}{$\begin{array}{l}\text { I+ year ago } \\
(n=293 \text { I0) }\end{array}$} & \multicolumn{2}{|c|}{$\begin{array}{l}\text { Last year } \\
(n=46220)\end{array}$} \\
\hline & No & $\%$ & No & $\%$ & No & $\%$ \\
\hline \multicolumn{7}{|l|}{ Sex } \\
\hline Male & 24453 & 50.3 & 14443 & 53.3 & 18303 & 43.7 \\
\hline Female & 27314 & 49.7 & 14867 & 46.7 & 27917 & 56.3 \\
\hline \multicolumn{7}{|l|}{ Age (years) } \\
\hline $12-24$ & 9085 & 20.7 & 6945 & 27.1 & 3999 & 11.9 \\
\hline $25-44$ & $|876|$ & 40.0 & 9873 & 36.9 & 7875 & 22.4 \\
\hline $45-64$ & $|834|$ & 33.0 & 8686 & 28.1 & 14929 & 34.7 \\
\hline $65+$ & 5580 & 6.2 & 3806 & 7.9 & 19417 & 31.0 \\
\hline \multicolumn{7}{|l|}{ Married status } \\
\hline Single & 16475 & 32.3 & |067| & 37.6 & 8415 & 20.1 \\
\hline Married/common law & 27161 & 57.9 & 13945 & 52.6 & 24866 & 63.1 \\
\hline Separated/divorced/widowed & 7997 & 9.8 & 4642 & 9.8 & $|285|$ & 16.8 \\
\hline Unknown & 134 & - & 52 & - & 88 & - \\
\hline \multicolumn{7}{|l|}{ Smoking status } \\
\hline Smoker & 13409 & 24.6 & 7555 & 24.8 & 7550 & 15.9 \\
\hline Former smoker & 18949 & 34.8 & 10671 & 34.0 & 21984 & 43.7 \\
\hline Non-smoker & 19247 & 40.6 & 10970 & 41.2 & 16505 & 40.4 \\
\hline Unknown & 162 & - & 114 & - & 181 & - \\
\hline \multicolumn{7}{|l|}{ Education } \\
\hline Low & 12748 & 21.2 & 7246 & 21.8 & 12898 & 24.4 \\
\hline Middle & 12330 & 25.0 & 6832 & 25.3 & 9128 & 21.1 \\
\hline High & 25477 & 53.9 & 14512 & 52.9 & 22904 & 54.5 \\
\hline Unknown & 212 & - & 720 & - & 1290 & - \\
\hline \multicolumn{7}{|l|}{ Income } \\
\hline Low & 14505 & 25.9 & 7492 & 23.5 & 16093 & 30.4 \\
\hline Middle & 15579 & 35.7 & 8202 & 32.9 & 12396 & 33.7 \\
\hline High & 14005 & 38.5 & 8859 & 43.6 & 10068 & 35.9 \\
\hline Unknown & 7678 & - & 4757 & - & 7663 & - \\
\hline \multicolumn{7}{|l|}{ Being immigrants } \\
\hline Immigrant & 6572 & 21.1 & 3657 & 20.1 & 7140 & 25.0 \\
\hline Non-immigrant & 43937 & 78.9 & 24951 & 79.9 & 37939 & 75.0 \\
\hline Unknown & 1258 & - & 702 & - & || $4 \mid$ & - \\
\hline \multicolumn{7}{|l|}{ Race } \\
\hline White & 43502 & 80.9 & 23891 & 79.2 & 39183 & 80.6 \\
\hline Non-White & 6545 & 19.1 & 4298 & 20.8 & 5170 & 19.4 \\
\hline Unknown & 1720 & - & $|12|$ & - & 1867 & - \\
\hline \multicolumn{7}{|l|}{ Physical activity } \\
\hline Active & $13 \mid 43$ & 25.4 & 8611 & 29.2 & 10643 & 24.1 \\
\hline Moderate & 12810 & 24.5 & 7269 & 24.5 & 11224 & 24.5 \\
\hline Inactive & 25744 & 50.1 & 13383 & 46.3 & 24291 & 51.4 \\
\hline Unknown & 70 & - & 47 & - & 62 & - \\
\hline \multicolumn{7}{|l|}{ Major disease } \\
\hline No & 44536 & 87.9 & 23436 & 81.8 & 29970 & 69.3 \\
\hline Yes & 7231 & 12.1 & 5874 & 18.2 & 16250 & 30.7 \\
\hline \multicolumn{7}{|l|}{ Non major disease } \\
\hline No & $2837 \mid$ & 60.1 & 14675 & 54.7 & 14509 & 38.2 \\
\hline Yes & 23396 & 39.9 & 14635 & 45.3 & 31711 & 61.8 \\
\hline \multicolumn{7}{|l|}{ Self-perceived health } \\
\hline Excellent & 11792 & 25.1 & 5658 & 21.3 & 6873 & 17.6 \\
\hline Very good & 20294 & 39.9 & 11364 & 38.9 & 15226 & 34.3 \\
\hline Good & 14802 & 27.4 & 8830 & 29.7 & 14965 & 31.8 \\
\hline Fair & 3773 & 6.0 & 2589 & 7.5 & 6715 & 12.1 \\
\hline
\end{tabular}


Table I (Continued)

\begin{tabular}{|c|c|c|c|c|c|c|}
\hline \multirow[t]{3}{*}{ Characteristics } & \multicolumn{6}{|c|}{ Influenza vaccination history } \\
\hline & \multicolumn{2}{|c|}{$\begin{array}{l}\text { Never } \\
(n=51767)\end{array}$} & \multicolumn{2}{|c|}{$\begin{array}{l}I+\text { year ago } \\
(n=293 I 0)\end{array}$} & \multicolumn{2}{|c|}{$\begin{array}{l}\text { Last year } \\
(n=46220)\end{array}$} \\
\hline & No & $\%$ & No & $\%$ & No & $\%$ \\
\hline Poor & 1045 & 1.6 & 833 & 2.5 & 2344 & 4.2 \\
\hline Unknown & 61 & - & 36 & - & 97 & - \\
\hline \multicolumn{7}{|l|}{ Provinces } \\
\hline Ontario & 38452 & 68.9 & 18012 & 54.1 & 27842 & 54.3 \\
\hline Other provinces/territories & 13315 & 31.1 & 11298 & 45.9 & 18378 & 45.7 \\
\hline
\end{tabular}

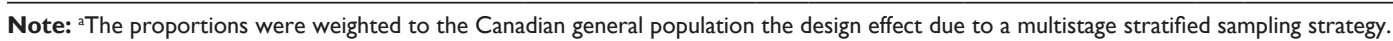

Table 2 Reasons for not having influenza vaccination in the past year by sex and flu shot history, the Canadian Community Health Survey 2007-2008

\begin{tabular}{|c|c|c|c|c|c|c|c|c|c|c|}
\hline \multirow[t]{3}{*}{ Reason } & \multicolumn{4}{|l|}{ Sex } & \multicolumn{4}{|c|}{$\begin{array}{l}\text { Influenza } \\
\text { vaccination history }\end{array}$} & & \\
\hline & \multicolumn{2}{|c|}{$\begin{array}{l}\text { Men } \\
(n=38896)\end{array}$} & \multicolumn{2}{|c|}{$\begin{array}{l}\text { Women } \\
(n=42 \mid 8 I)\end{array}$} & \multicolumn{2}{|c|}{$\begin{array}{l}\text { Never } \\
(n=5 \mid 767)\end{array}$} & \multicolumn{2}{|c|}{$\begin{array}{l}\text { I+ year ago } \\
(n=29310)\end{array}$} & \multicolumn{2}{|c|}{$\begin{array}{l}\text { Total } \\
(\mathrm{n}=8 \text { I 077) }\end{array}$} \\
\hline & No & $\%^{a}$ & No & $\%^{\mathrm{a}}$ & No & $\%^{\mathrm{a}}$ & No & $\%^{\mathrm{a}}$ & No & $\%^{\mathrm{a}}$ \\
\hline Have not gotten around to it & 6958 & 19.5 & 6143 & 15.6 & 4864 & 10.3 & 8237 & 31.0 & $1310 \mid$ & 17.6 \\
\hline Respondent did not think necessary & 27173 & 72.2 & 28272 & 70.3 & 42223 & 83.4 & 13222 & 49.0 & 55445 & 71.3 \\
\hline Doctor did not think necessary & 659 & 1.8 & $|27|$ & 2.9 & 1353 & 2.5 & 577 & 1.9 & 1930 & 2.3 \\
\hline Personal/family responsibility & 236 & 0.6 & 265 & 0.7 & 149 & 0.3 & 352 & 1.3 & 501 & 0.7 \\
\hline Not available at time when required & 407 & 1.1 & 440 & 1.0 & 159 & 0.3 & 688 & 2.3 & 847 & 1.0 \\
\hline Not available at all in area & 123 & 0.3 & 170 & 0.4 & 83 & 0.1 & 210 & 0.6 & 293 & 0.3 \\
\hline Waiting time was too long & 75 & 0.2 & 87 & 0.2 & 67 & 0.1 & 95 & 0.4 & 162 & 0.2 \\
\hline Transportation problems & 30 & 0.1 & 68 & 0.2 & 27 & 0.1 & 71 & 0.2 & 98 & 0.1 \\
\hline Language problem & 6 & 0.1 & 9 & 0.0 & 11 & 0.1 & 4 & 0.0 & 15 & 0.1 \\
\hline Cost & 195 & 0.5 & 288 & 0.8 & 271 & 0.6 & 212 & 0.7 & 483 & 0.6 \\
\hline Did not know where to go & 391 & 1.1 & 323 & 0.9 & 355 & 0.9 & 359 & 1.2 & 714 & 1.0 \\
\hline Fear & 1212 & 3.1 & 2446 & 5.2 & 2844 & 4.8 & 814 & 2.8 & 3658 & 4.1 \\
\hline Bad reaction to previous shot & 1847 & 4.5 & 3124 & 6.2 & 697 & 1.1 & 4274 & 13.2 & 4971 & 5.3 \\
\hline Unable leave house/health & 42 & 0.1 & 138 & 0.3 & 34 & 0.0 & 146 & 0.4 & 180 & 0.2 \\
\hline Other & 344 & 0.8 & 532 & 1.4 & 270 & 0.5 & 606 & 2.1 & 876 & I.I \\
\hline
\end{tabular}

Note: ${ }^{2}$ The proportions were weighted to the Canadian general population.

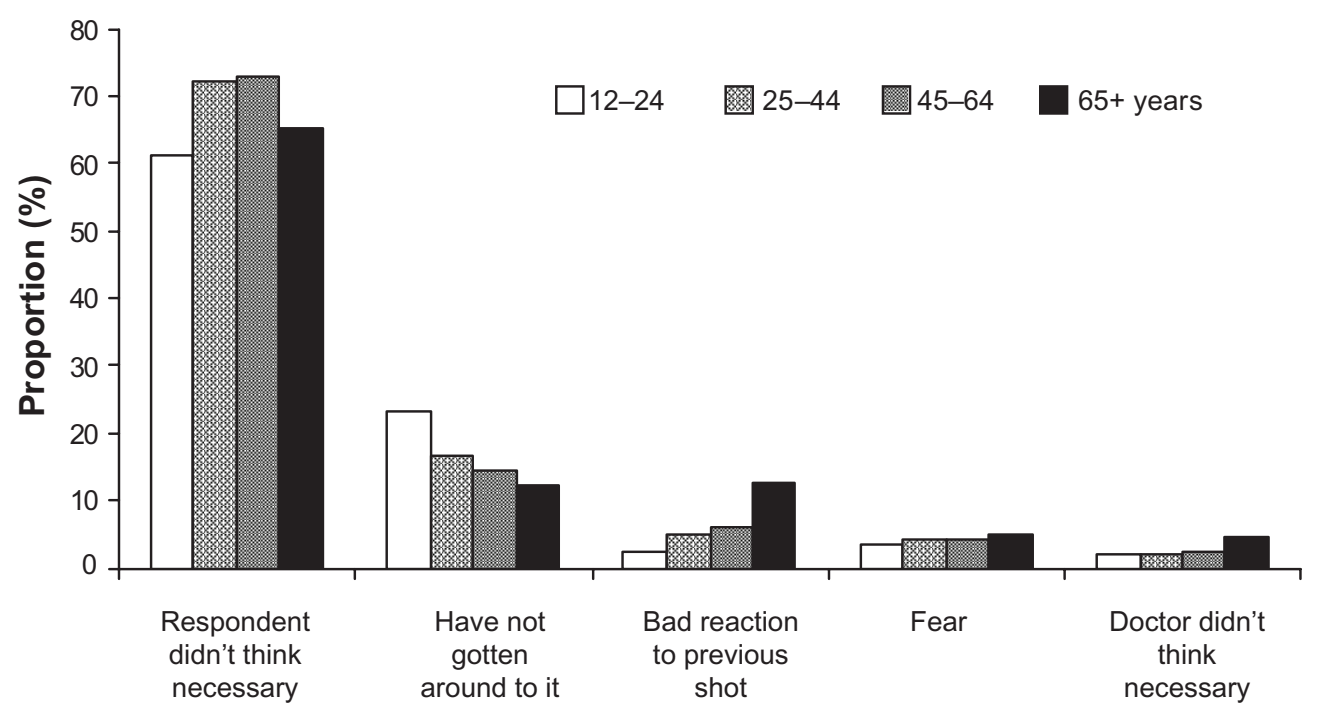

Reasons for not having influenza vaccination

Figure I Reasons for not have influenza vaccination during the past year by age, Canadian Community Health Survey 2007-2008. 
Table 3 Adjusted relative risks (95\% confidence intervals) for reasons for not having influenza vaccination during the past year associated with various factors, Canadian Community Health Survey 2007-2008

\begin{tabular}{|c|c|c|c|c|c|}
\hline & $\begin{array}{l}\text { Respondent did } \\
\text { not think necessary }\end{array}$ & $\begin{array}{l}\text { Have not gotten } \\
\text { around to it }\end{array}$ & $\begin{array}{l}\text { Bad reaction to } \\
\text { previous shot }\end{array}$ & Fear & $\begin{array}{l}\text { Doctor did not } \\
\text { think necessary }\end{array}$ \\
\hline \multicolumn{6}{|l|}{ Sex } \\
\hline Male & 1.00 (reference) & I.00 (reference) & I.00 (reference) & I.00 (reference) & 1.00 (reference) \\
\hline Female & $0.98(0.97,0.99)$ & $0.84(0.81,0.88)$ & $1.33(1.23,1.43)$ & $1.66(1.53,1.82)$ & $1.51(1.34,1.70)$ \\
\hline \multicolumn{6}{|l|}{ Age (years) } \\
\hline $12-24$ & $0.90(0.87,0.92)$ & $1.90(1.73,2.09)$ & $0.31(0.26,0.37)$ & & $0.61(0.50,0.76)$ \\
\hline $25-44$ & $0.98(0.96,1.00)$ & I.43 (I.30, I.58) & $0.57(0.50,0.64)$ & & $0.53(0.44,0.66)$ \\
\hline $45-64$ & $1.02(1.00,1.04)$ & $1.29(1.17,1.42)$ & $0.58(0.52,0.64)$ & & $0.68(0.57,0.83)$ \\
\hline $65+$ & 1.00 (reference) & 1.00 (reference) & 1.00 (reference) & & 1.00 (reference) \\
\hline \multicolumn{6}{|l|}{ Married status } \\
\hline Single & 1.00 (reference) & & I.00 (reference) & & \\
\hline Married/common law & $1.01(1.00,1.03)$ & & $1.12(1.00,1.26)$ & & \\
\hline $\begin{array}{l}\text { Separated/divorced/ } \\
\text { widowed }\end{array}$ & I.0I $(0.99,1.03)$ & & $1.22(1.06,1.41)$ & & \\
\hline \multicolumn{6}{|l|}{ Smoking status } \\
\hline Smoker & & $1.09(1.03,1.13)$ & $1.16(1.05,1.28)$ & $1.17(1.05,1.30)$ & $0.67(0.57,0.79)$ \\
\hline Former smoker & & $1.06(1.01,1.11)$ & $1.05(0.96,1.15)$ & $1.09(0.99,1.20)$ & $0.85(0.74,0.97)$ \\
\hline Non-smoker & & 1.00 (reference) & I.00 (reference) & I.00 (reference) & 1.00 (reference) \\
\hline \multicolumn{6}{|l|}{ Education } \\
\hline Low & 1.00 (reference) & I.00 (reference) & & & 1.00 (reference) \\
\hline Middle & $1.03(1.02,1.05)$ & $1.15(1.09,1.21)$ & & & $0.67(0.56,0.80)$ \\
\hline High & $1.03(1.02,1.05)$ & $1.06(1.00,1.12)$ & & & $0.81(0.69,0.94)$ \\
\hline \multicolumn{6}{|l|}{ Income } \\
\hline Low & 1.00 (reference) & & & & \\
\hline Middle & $1.01(1.00,1.03)$ & & & & \\
\hline High & $1.00(0.98,1.01)$ & & & & \\
\hline \multicolumn{6}{|l|}{ Being immigrants } \\
\hline Immigrant & $0.98(0.97,1.00)$ & & & & \\
\hline Non-immigrant & 1.00 (reference) & & & & \\
\hline \multicolumn{6}{|l|}{ Race } \\
\hline White & $1.04(1.03,1.06)$ & $0.92(0.88,0.96)$ & I.3I (I.18, I.47) & & \\
\hline Non-White & 1.00 (reference) & 1.00 (reference) & 1.00 (reference) & & \\
\hline \multicolumn{6}{|l|}{ Physical activity } \\
\hline Active & 1.00 (reference) & I.00 (reference) & I.00 (reference) & & \\
\hline Moderate & $1.01(1.00,1.02)$ & $1.04(0.99,1.10)$ & $0.84(0.76,0.94)$ & & \\
\hline Inactive & $0.98(0.97,0.99)$ & $1.16(1.11,1.21)$ & $0.77(0.7 \mathrm{I}, 0.85)$ & & \\
\hline \multicolumn{6}{|l|}{ Major disease } \\
\hline No & 1.00 (reference) & 1.00 (reference) & I.00 (reference) & I.00 (reference) & 1.00 (reference) \\
\hline Yes & $0.92(0.91,0.94)$ & $1.09(1.04,1.15)$ & $1.50(1.37,1.64)$ & $1.26(1.13,1.41)$ & $1.25(1.08,1.46)$ \\
\hline \multicolumn{6}{|l|}{ Non major disease } \\
\hline No & 1.00 (reference) & 1.00 (reference) & I.00 (reference) & I.00 (reference) & 1.00 (reference) \\
\hline Yes & $0.97(0.96,0.98)$ & $1.04(1.00,1.08)$ & $1.47(1.35,1.59)$ & $1.21(1.11,1.33)$ & $1.23(1.09,1.40)$ \\
\hline \multicolumn{6}{|l|}{ Self-perceived health } \\
\hline Excellent & 1.00 (reference) & 1.00 (reference) & I.00 (reference) & I.00 (reference) & 1.00 (reference) \\
\hline Very good & $0.98(0.97,0.99)$ & $1.08(1.03,1.14)$ & $1.00(0.90,1.11)$ & $1.15(1.02,1.30)$ & $0.98(0.84,1.15)$ \\
\hline Good & $0.96(0.94,0.97)$ & $1.05(1.00,1.11)$ & $1.17(1.05,1.31)$ & $1.40(1.23,1.59)$ & $1.12(0.95,1.33)$ \\
\hline Fair & $0.93(0.91,0.95)$ & $1.12(1.03,1.21)$ & $1.34(1.16,1.56)$ & $1.55(1.30,1.85)$ & $1.28(1.00,1.61)$ \\
\hline Poor & $0.82(0.77,0.86)$ & $0.95(0.82,1.10)$ & $1.55(1.27,1.89)$ & $1.90(1.47,2.43)$ & $1.61(1.14,2.22)$ \\
\hline \multicolumn{6}{|l|}{ Provinces } \\
\hline Ontario & $0.97(0.96,0.98)$ & $1.19(1.15,1.24)$ & $1.56(1.45,1.68)$ & $1.63(1.50,1.78)$ & $1.37(122,1.54)$ \\
\hline $\begin{array}{l}\text { Other provinces/ } \\
\text { territories }\end{array}$ & 1.00 (reference) & 1.00 (reference) & 1.00 (reference) & 1.00 (reference) & 1.00 (reference) \\
\hline \multicolumn{6}{|c|}{ History of influenza vaccination } \\
\hline No & 1.00 (reference) & I.00 (reference) & & I.00 (reference) & 1.00 (reference) \\
\hline Yes & $0.59(0.58,0.59)$ & $2.73(2.63,2.85)$ & & $0.5 \mathrm{I}(0.46,0.56)$ & $0.66(0.58,0.75)$ \\
\hline
\end{tabular}




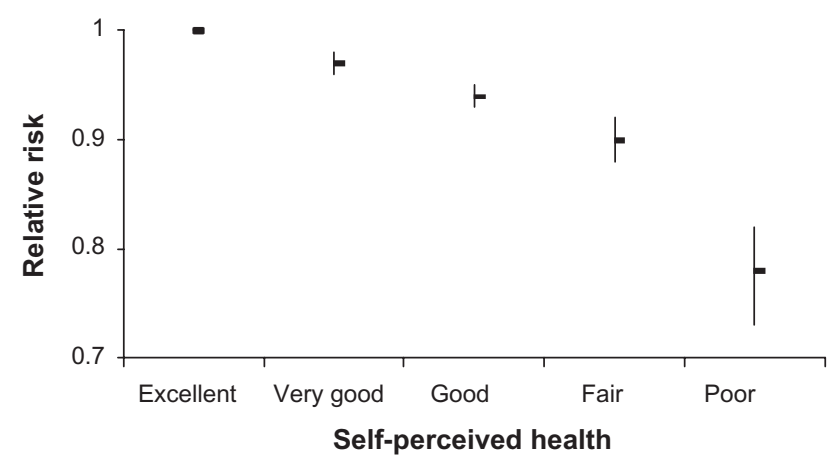

Figure $\mathbf{2}$ "Respondent did not think necessary" as a reason for not having influenza vaccination during the past year associated with self-perceived health after excluding chronic disease, Canadian Community Health Survey 2007-2008.

associated with "Respondent did not think it necessary" as a reason for not having an influenza vaccination in the past year and healthy people were more likely to report this reason, and relatively less likely to report other reasons. For those who had influenza vaccination previously, the main reason for not having an influenza vaccination in the last year was not having gotten around to it.

Table 4 (Appendix 1 for full models) shows the proportions of various reasons for reporting not having received an influenza vaccination during the past 12 months in Ontario and other regions. After adjustment for covariates, Ontarians were less likely to report "Respondent did not think it necessary" (adjusted RR: 0.97, 95\% CI: 0.96, 0.98), but more likely to report "Have not gotten around to it" (adjusted RR:

Table 4 Reasons for not having influenza vaccination shot during the past year in Ontario and other regions, the Canadian Community Health Survey 2007-2008

\begin{tabular}{|c|c|c|c|c|}
\hline \multirow[t]{2}{*}{ Reason } & \multicolumn{2}{|c|}{$\begin{array}{l}\text { Ontario } \\
(n=246 \mid 3)\end{array}$} & \multicolumn{2}{|c|}{$\begin{array}{l}\text { Other } \\
(n=56464)\end{array}$} \\
\hline & No & $\%^{\mathrm{a}}$ & No & $\%^{a}$ \\
\hline Have not gotten around to it & 4807 & 21.6 & 8294 & 15.3 \\
\hline Respondent did not think necessary & 15535 & 65.7 & 39910 & 74.5 \\
\hline Doctor did not think necessary & 674 & 2.7 & 1256 & 2.1 \\
\hline Personal/family responsibility & 190 & 0.8 & 311 & 0.6 \\
\hline Not available when required & 323 & $\mathrm{I} .4$ & 524 & 0.8 \\
\hline Not available in area & 89 & 0.3 & 204 & 0.3 \\
\hline Waiting time was too long & 66 & 0.3 & 96 & 0.2 \\
\hline Transportation problems & 34 & 0.1 & 64 & 0.1 \\
\hline Language problem & 5 & 0.1 & 10 & 0.0 \\
\hline Cost & 27 & 0.1 & 456 & 0.9 \\
\hline Did not know where to go & 172 & 0.7 & 542 & 1.2 \\
\hline Fear & 1402 & 5.2 & 2256 & 3.5 \\
\hline Bad reaction to previous shot & 1935 & 6.8 & 3036 & 4.5 \\
\hline Unable leave house/health & 66 & 0.2 & 114 & 0.2 \\
\hline Other & 313 & 1.3 & 563 & 1.0 \\
\hline
\end{tabular}

Note: ${ }^{2}$ The proportions were weighted to the Canadian general population.
$1.19,95 \%$ CI: $1.15,1.24)$, "Bad reaction to previous shot" (adjusted RR: 1.56, 95\% CI: 1.45, 1.68), "Fear" (adjusted RR: $1.63,95 \%$ CI: 1.50, 1.78), and "Doctor did not think it necessary” (adjusted RR: 1.37, 95\% CI: 1.22, 1.54).

\section{Discussion}

Our data demonstrated that in Canada, individuals who were male or young had a lower rate and those who had a chronic condition or poor self-perceived health had a higher rate of seasonal influenza vaccination. The most common reason for not having an influenza vaccination in the past year is that respondents did not think it was necessary, and this was more so in those who had never been immunized against influenza (83\%) compared to those who had (49\%). There was a notable age difference that children and youth as compared with adults were more likely to think a seasonal vaccination unnecessary compared with others. The results were comparable to previous findings observed in children ${ }^{11}$ and youth and the elderly. ${ }^{8,9,12}$ Those who had chronic disease and self-perceived poor health were less likely to think an influenza vaccination unnecessary, which echoes some previous results that having a chronic disease and self-perceived health status are important predictors for uptake of influenza vaccination..$^{13}$ The association between self-perceived health and belief that an influenza vaccination is unnecessary showed a linear trend in that those who had poorer self-perceived health were less likely to have this belief that influenza vaccination is unnecessary. Ontario has established the Universal Influenza Immunization Program, which provides free influenza vaccination to all residents excluding neonates and has promoted the program since 2000. The data demonstrated that Ontarians were less likely to report that influenza vaccination was unnecessary as a reason for not having an influenza vaccination compared with other regions. More jurisdictions have had these programs since the survey was conducted and the situation might have changed.

The second most common reason for reporting not having received the influenza vaccination was that respondents had not gotten around to it. Male sex, young age, smoking, physical inactivity, and non-White race were associated with an increased likelihood of reporting this reason. Immunization sites may not be open at the right time for some sub-groups. There may be prioritization issues in terms of time management for individuals with different characteristics. We noticed that those who reported having had influenza vaccination previously were substantially more likely to report that 
they had not gotten around to having influenza vaccination compared to those who reported having no influenza vaccination history and Ontarians, with a large urban population, had a higher proportion of this response than people in other provinces.

Bad reaction to the previous influenza vaccination was the third most important reason for not having influenza vaccination in the previous year. The seasonal influenza vaccine in general is very safe and only a small number of people are reported to have some mild side effects after influenza vaccination, ${ }^{14,15}$ such as crankiness, irritability, general malaise, soreness and pain, allergic reactions, mild fever, nausea, and headaches. There is a rare occurrence of neurological complications such as Guillain-Barré syndrome after influenza vaccination. ${ }^{16-19}$ The proportion of individuals reporting not having received the influenza vaccine because they reported having had a previous bad reaction was higher in Ontario than other regions as a whole, which might be due to a higher vaccination rate in Ontario. Living in Ontario was associated with an approximately $50 \%$ increase in influenza vaccination rate during the $2007 / 2008$ season [odds ratio (OR) 1.50, 95\% confidence interval (CI) 1.45-1.55]. ${ }^{20}$

Approximately $4 \%$ and $2 \%$ of those who had no influenza vaccination in the previous year reported "Fear" and "Doctor did not think it necessary." Female participants and those with chronic poorer health were more likely to report these reasons. People with chronic conditions were more likely to consult with physicians, which might increase the likelihood of obtaining the opinion from medical doctors. Not all physicians think that influenza vaccination is effective and/or necessary for all the people.

In Canada, at the time that the survey was conducted, Ontario was the only province that provided free influenza vaccination to its residents of all ages. In a previous study conducted in the United States, ${ }^{21}$ one-third of vaccine recipients would refuse vaccination if asked to pay at least $\$ 10$. Cost may be a primary reason for low coverage in Poland. ${ }^{22}$ However, the affordability of the vaccine in general is not likely an important issue in Canada, and only less than 1 percent reported cost as a reason for not getting vaccinated.

The study has some limitations. The national survey did not include children under the age of 12 years and those living on Indian Reserves or Crown lands and residents of institutions, who may be at high risk of influenza infections. Information misclassification clearly existed, for example, some respondents (1\%) who had not had influenza vaccination reported having a bad reaction to a previous one. Non-respondents accounted for about one quarter of the selected population. There might be considerable recall bias. We are not able to provide a reasonable estimation for the size of the bias. The analysis was based on existing data collected from Statistics Canada and some important answers to the question, "What are the reasons that you have not had a flu shot in the past year?" such as "worry about the adverse reaction" and "the flu shot makes me sick," were not included in the questionnaire.

It is important to understand the factors influencing the decision to receive a seasonal influenza vaccination, which will be helpful to guide the development and improvement of influenza vaccination programs for the general population. Since the vast majority of people who did not receive an influenza vaccination during the past year thought it was not necessary, it would be crucial to increase awareness of the importance of influenza vaccination among these people, especially those who perceived themselves to be healthy. More and stronger evidence for the effectiveness and personal/population benefits of seasonal influenza vaccination would be most helpful to motivate people to have the vaccination and improve uptake among the general population. Male and younger participants were more likely to report, "Have not gotten around to it" than female and older ones. Issues related to programmatic implementation of vaccine program need to be further examined and addressed to make flu shots easier to access for various subpopulations.

\section{Disclosure}

The authors report no conflicts of interest in this work.

\section{References}

1. Monto AS. Seasonal influenza and vaccination coverage. Vaccine. 2010;28 Suppl 4:D33-D44.

2. Kwong JC, Stukel TA, Lim J, McGeer AJ, Upshur RE, Johansen H, et al. The effect of universal influenza immunization on mortality and health care use. PLoS Med. 2008;5(10):e211.

3. Campitelli MA, Rosella LC, Stukel TA, Kwong JC. Influenza vaccination and all-cause mortality in community-dwelling elderly in Ontario, Canada, a cohort study. Vaccine. 2010;29(2):240-246.

4. Chen Y, Wu J, Yi QL. Reduced risk of hospitalization associated with influenza vaccination in Canada. Vaccine. 2010;28(11):2290-2295.

5. Hoen AG, Buckeridge DL, Charland KM, Mandl KD, Quach C, Brownstein JS. Effect of expanded US recommendations for seasonal influenza vaccination: comparison of two pediatric emergency departments in the United States and Canada. CMAJ. 2011;183(13):E1025-E1032.

6. Kwong JC, Ge H, Rosella LC, Guan J, Maaten S, Moran K, et al. School-based influenza vaccine delivery, vaccination rates, and healthcare use in the context of a universal influenza immunization program: an ecological study. Vaccine. 2010;28(15):2722-2729.

7. Statement on influenza vaccination for the 2007-2008 season. An Advisory Committee Statement (ACS). Can Commun Dis Rep. 2007; 33(ACS-7): 1-38.

8. Johansen H, Sambell C, Zao X. Flu shots - National and provincial/ territorial trends. Health Rep. 2006;17(2):43-48. 
9. Kwong JC, Rosella LC, Johansen H. Trends in influenza vaccination in Canada, 1996/1997 to 2005. Health Rep. 2007;18(4):9-19.

10. Chen Y, Dales R, Krewski D, Breithaupt K. Increased effects of smoking and obesity on asthma among female Canadians: the National Population Health Survey, 1994-1995. Am J Epidemiol. 1999;150(3):255-262.

11. Li Z, Doan Q, Dobson S. Determinants of influenza immunization uptake in Canadian youths. Vaccine. 2009;28(19):3462-3466.

12. Influenza vaccination and self-reported reasons for not receiving influenza vaccination among Medicare beneficiaries aged $\geq 65$ years United States, 1991-2002. MMWR Morb Mortal Wkly Rep. 2004;53(43): 1012-1015.

13. Chen Y, Yi QL, Wu J, Li F. Chronic disease status, self-perceived health and hospital admissions are important predictors for having a flu shot in Canada. Vaccine. 2007;25(42):7436-7440.

14. Jefferson T, Di Pietrantonj C, Rivetti A, Bawazeer GA, Al-Ansary LA, Ferroni E. Vaccines for preventing influenza in healthy adults. Cochrane Database Syst Rev. 2010;7:CD001269.

15. Jefferson TO, Rivetti D, Di Pietrantonj C, Rivetti A, Demicheli V. Vaccines for preventing influenza in healthy adults. Cochrane Database Syst Rev. 2007;2:CD001269.

16. Bedard Marrero V, Osorio Figueroa RL, Vazquez Torres O. Guillain-Barre syndrome after influenza vaccine administration: two adult cases. Bol Asoc Med P R. 2010;102(2):39-41.
17. Lasky T, Terracciano GJ, Magder L, Koski CL, Ballesteros M, Nash D, et al. The Guillain-Barre syndrome and the 1992-1993 and 1993-1994 influenza vaccines. N Engl J Med. 1998;339(25):1797-1802.

18. Nakamura N, Nokura K, Zettsu T, Koga H, Tachi M, Terada M, et al. Neurologic complications associated with influenza vaccination: two adult cases. Intern Med. 2003;42(2):191-194.

19. Kao CD, Chen JT, Lin KP, Shan DE, Wu ZA, Liao KK. Guillain-Barre syndrome coexisting with pericarditis or nephrotic syndrome after influenza vaccination. Clin Neurol Neurosurg. 2004;106(2):136-138.

20. Polisena J, Chen Y, Manuel D. The proportion of influenza vaccination in Ontario, Canada in 2007/2008 compared with other provinces. Vaccine. 2012;30(11):1981-1985.

21. Steiner M, Vermeulen LC, Mullahy J, Hayney MS. Factors influencing decisions regarding influenza vaccination and treatment: a survey of healthcare workers. Infect Control Hosp Epidemiol. 2002;23(10):625-627.

22. Kardas P, Zasowska A, Dec J, Stachurska M. Reasons for low influenza vaccination coverage: cross-sectional survey in Poland. Croat Med J. 2011;52(2):126-133. 


\section{Appendix table}

Appendix I Adjusted relative risks (95\% confidence intervals) for reasons not having influenza vaccination during the past year associated with various factors, Canadian Community Health Survey 2007-2008 - full models

\begin{tabular}{|c|c|c|c|c|c|}
\hline & $\begin{array}{l}\text { Respondent did } \\
\text { not think necessary }\end{array}$ & $\begin{array}{l}\text { Have not gotten } \\
\text { around to it }\end{array}$ & $\begin{array}{l}\text { Bad reaction to } \\
\text { previous shot }\end{array}$ & Fear & $\begin{array}{l}\text { Doctor did not } \\
\text { think necessary }\end{array}$ \\
\hline \multicolumn{6}{|l|}{ Sex } \\
\hline Male & I.00 (reference) & I.00 (reference) & I.00 (reference) & $\mathrm{I} .00$ (reference) & I.00 (reference) \\
\hline Female & $0.98(0.97,0.99)$ & $0.84(0.8 \mathrm{I}, 0.87)$ & $1.32(1.23,1.43)$ & $1.66(1.52, I .8 \mathrm{I})$ & I.5I (I.34, I.7I) \\
\hline \multicolumn{6}{|l|}{ Age (years) } \\
\hline $12-24$ & $0.90(0.87,0.92)$ & $1.91(1.71,2.13)$ & $0.30(0.25,0.37)$ & $0.85(0.69,1.06)$ & $0.66(0.50,0.87)$ \\
\hline $25-44$ & $0.98(0.96,1.01)$ & $1.43(1.29,1.58)$ & $0.54(0.48,0.6 \mathrm{I})$ & $0.94(0.79,1.12)$ & $0.54(0.44,0.68)$ \\
\hline $45-64$ & $1.02(1.00,1.04)$ & $1.28(1.16,1.41)$ & $0.56(0.50,0.62)$ & $0.87(0.74,1.04)$ & $0.68(0.56,0.83)$ \\
\hline $65+$ & I.00 (reference) & 1.00 (reference) & I.00 (reference) & 1.00 (reference) & 1.00 (reference) \\
\hline \multicolumn{6}{|l|}{ Married status } \\
\hline Single & I.00 (reference) & I.00 (reference) & I.00 (reference) & 1.00 (reference) & I.00 (reference) \\
\hline Married/common law & $1.01(1.00,1.03)$ & $1.02(0.97,1.08)$ & $1.12(1.00,1.26)$ & $0.99(0.88,1.12)$ & $1.10(0.92,1.32)$ \\
\hline $\begin{array}{l}\text { Separated/divorced/ } \\
\text { widowed }\end{array}$ & $1.01(0.99,1.03)$ & $1.03(0.95,1.12)$ & $1.24(1.08,1.43)$ & $0.87(0.73,1.04)$ & $1.22(0.96,1.55)$ \\
\hline Unknown & $0.93(0.79,1.04)$ & $0.96(0.55,1.47)$ & I. $17(0.44,2.39)$ & $1.60(0.65,3.13)$ & I.46 $(0.3 \mathrm{I}, 4.00)$ \\
\hline \multicolumn{6}{|l|}{ Smoking status } \\
\hline Smoker & $0.99(0.98,1.00)$ & $1.08(1.03,1.14)$ & $1.16(1.05,1.28)$ & $1.13(1.01,1.26)$ & $0.68(0.57,0.80)$ \\
\hline Former smoker & $1.00(0.99,1.01)$ & $1.05(1.01,1.10)$ & $1.04(0.95,1.14)$ & $1.07(0.97,1.19)$ & $0.85(0.74,0.97)$ \\
\hline Non-smoker & I.00 (reference) & 1.00 (reference) & I.00 (reference) & 1.00 (reference) & 1.00 (reference) \\
\hline Unknown & $0.99(0.88,1.06)$ & $0.98(0.69,1.33)$ & $1.22(0.69,1.98)$ & $0.83(0.35,1.63)$ & $0.67(0.23,1.52)$ \\
\hline \multicolumn{6}{|l|}{ Education } \\
\hline Low & I.00 (reference) & I.00 (reference) & I.00 (reference) & I.00 (reference) & I.00 (reference) \\
\hline Middle & $1.03(1.02,1.05)$ & $1.15(1.09,1.21)$ & $1.03(0.92,1.16)$ & $0.93(0.82,1.05)$ & $0.67(0.56,0.80)$ \\
\hline High & $1.03(1.02,1.05)$ & $1.05(1.00,1.11)$ & $1.13(1.02,1.26)$ & $0.96(0.86,1.09)$ & $0.80(0.69,0.94)$ \\
\hline Unknown & $1.02(0.97,1.07)$ & $1.10(0.89,1.37)$ & $1.05(0.68,1.56)$ & $0.85(0.5 \mathrm{I}, \mathrm{I} .37)$ & $0.67(0.35,1.22)$ \\
\hline \multicolumn{6}{|l|}{ Income } \\
\hline Low & I.00 (reference) & I.00 (reference) & I.00 (reference) & I.00 (reference) & I.00 (reference) \\
\hline Middle & I.0I (1.00, I.03) & $0.95(0.90,1.00)$ & I.I0 (0.99, I.23) & $0.96(0.85,1.08)$ & I.0I $(0.86,1.20)$ \\
\hline High & $0.99(0.98,1.01)$ & $1.04(0.98,1.10)$ & $1.03(0.92,1.16)$ & $0.88(0.78,1.00)$ & I. $15(0.97,1.37)$ \\
\hline Unknown & $0.96(0.94,0.98)$ & $0.97(0.91,1.04)$ & $1.15(1.01,1.30)$ & $1.04(0.90,1.19)$ & I.08 $(0.89,1.3 \mathrm{I})$ \\
\hline \multicolumn{6}{|l|}{ Being immigrants } \\
\hline Immigrant & $0.98(0.97,1.00)$ & $0.97(0.92,1.02)$ & $0.87(0.78,0.96)$ & $0.8 \mathrm{I}(0.7 \mathrm{I}, 0.92)$ & $0.98(0.83,1.15)$ \\
\hline Non-immigrant & I.00 (reference) & I.00 (reference) & I.00 (reference) & 1.00 (reference) & I.00 (reference) \\
\hline Unknown & $0.99(0.94,1.04)$ & $0.94(0.75,1.15)$ & $0.72(0.46, \mathrm{I} . \mathrm{II})$ & $0.98(0.6 \mathrm{I}, \mathrm{I} .52)$ & $1.33(0.73,2.26)$ \\
\hline \multicolumn{6}{|l|}{ Race } \\
\hline White & $1.04(1.03,1.06)$ & $0.90(0.86,0.95)$ & $1.22(1.08,1.38)$ & $1.01(0.89,1.15)$ & $0.98(0.82,1.16)$ \\
\hline Non-White & I.00 (reference) & I.00 (reference) & I.00 (reference) & 1.00 (reference) & I.00 (reference) \\
\hline Unknown & I.0I $(0.97,1.06)$ & $0.89(0.77,1.03)$ & $1.57(1.18,2.06)$ & $\mathrm{I} .04(0.72, \mathrm{I} .46)$ & $1.10(0.68,1.72)$ \\
\hline \multicolumn{6}{|l|}{ Physical activity } \\
\hline Active & I.00 (reference) & I.00 (reference) & I.00 (reference) & 1.00 (reference) & I.00 (reference) \\
\hline Moderate & I.0I $(0.99,1.02)$ & $1.04(0.99,1.10)$ & $0.85(0.76,0.94)$ & $0.92(0.8 \mathrm{I}, \mathrm{I} .04)$ & $0.87(0.74,1.03)$ \\
\hline Inactive & $0.98(0.97,0.99)$ & $1.16(1.11,1.22)$ & $0.78(0.7 \mathrm{I}, 0.85)$ & $0.96(0.86,1.07)$ & $0.97(0.84, I .12)$ \\
\hline Unknown & $0.96(0.82,1.06)$ & I.04 (0.5।, I.79) & $0.25(0.02,0.96)$ & $0.59(0.07,1.99)$ & $1.15(0.21,3.37)$ \\
\hline \multicolumn{6}{|l|}{ Major disease } \\
\hline No & I.00 (reference) & I.00 (reference) & I.00 (reference) & $\mathrm{I} .00$ (reference) & I.00 (reference) \\
\hline Yes & $0.92(0.91,0.94)$ & $1.09(1.04,1.15)$ & $1.49(1.37,1.63)$ & $1.23(1.10,1.38)$ & $1.25(1.08,1.46)$ \\
\hline \multicolumn{6}{|l|}{ Non major disease } \\
\hline No & I.00 (reference) & I.00 (reference) & I.00 (reference) & $\mathrm{I} .00$ (reference) & I.00 (reference) \\
\hline Yes & $0.97(0.96,0.98)$ & $1.04(1.00,1.08)$ & I.47 (I.35, I.59) & $1.21(1.10,1.32)$ & $1.23(1.09,1.40)$ \\
\hline \multicolumn{6}{|l|}{ Self-perceived health } \\
\hline Excellent & I.00 (reference) & I.00 (reference) & I.00 (reference) & I.00 (reference) & I.00 (reference) \\
\hline Very good & $0.98(0.97,0.99)$ & $1.08(1.03,1.14)$ & $\mathrm{I} .00(0.90, \mathrm{I} . \mathrm{II})$ & $1.15(1.02,1.30)$ & $0.99(0.84, I .16)$ \\
\hline Good & $0.96(0.94,0.97)$ & $1.06(1.00,1.12)$ & $1.19(1.06,1.33)$ & $1.40(1.24,1.60)$ & I.I4 (0.96, I.35) \\
\hline
\end{tabular}


Appendix I (Continued)

\begin{tabular}{|c|c|c|c|c|c|}
\hline & $\begin{array}{l}\text { Respondent did } \\
\text { not think necessary }\end{array}$ & $\begin{array}{l}\text { Have not gotten } \\
\text { around to it }\end{array}$ & $\begin{array}{l}\text { Bad reaction to } \\
\text { previous shot }\end{array}$ & Fear & $\begin{array}{l}\text { Doctor did not } \\
\text { think necessary }\end{array}$ \\
\hline Fair & $0.93(0.91,0.95)$ & $1.12(1.04,1.22)$ & $1.38(1.19,1.60)$ & $1.55(1.29,1.85)$ & $1.30(1.02,1.65)$ \\
\hline Poor & $0.82(0.77,0.86)$ & $0.96(0.83,1.11)$ & $1.61(1.31,1.96)$ & $1.93(1.48,2.47)$ & $1.63(1.15,2.25)$ \\
\hline Unknown & $0.82(0.59,1.02)$ & $1.07(0.37,2.17)$ & $1.73(0.59,3.55)$ & $1.22(0.14,4.10)$ & $1.20(0.09,4.66)$ \\
\hline \multicolumn{6}{|l|}{ Ontario } \\
\hline No & 1.00 (reference) & 1.00 (reference) & I.00 (reference) & I.00 (reference) & $\mathrm{I} .00$ (reference) \\
\hline Yes & $0.97(0.96,0.98)$ & $1.19(1.15,1.23)$ & $1.57(1.46,1.69)$ & $1.69(1.55,1.85)$ & $1.36(1.20,1.53)$ \\
\hline \multicolumn{6}{|c|}{ Flu shot history } \\
\hline No & I.00 (reference) & 1.00 (reference) & & I.00 (reference) & I.00 (reference) \\
\hline Yes & $0.59(0.58,0.60)$ & $2.73(2.62,2.84)$ & & $0.5 \mathrm{I}(0.46,0.56)$ & $0.66(0.58,0.75)$ \\
\hline
\end{tabular}

Vaccine: Development and Therapy

\section{Publish your work in this journal}

Vaccine: Development and Therapy is an international, peer-reviewed, open access journal that spans the spectrum of vaccine design and development through to clinical applications. The journal is characterized by the rapid reporting of application notes, reviews, original research and clinical studies in all therapeutic areas. Clinical outcomes, patient safety, and programs for the development and effective, safe, and sustained use of vaccines will be a feature of the journal. The manuscript management system is completely online and includes a very quick and fair peer-review system. Visit http://www.dovepress.com/testimonials.php to read real quotes from published authors.

Submit your manuscript here: http://www.dovepress.com/vaccine-development-and-therapy-journal 